\title{
Efficacy and Safety of Intra-Articular Hyaluronic Acid Injection (NRD101 / SUVENYL®) in Patients With Knee Osteoarthritis: a Multicenter, Randomized, Double-blind, Parallel, Active Control, Non-Inferiority Clinical Study
}

\section{Haoyang Wang}

West China Hospital of Medicine: Sichuan University West China Hospital

Wei Wang

Peking Union Medical College Hospital

\section{Yongsheng Xu}

Inner Mongolia People's Hospital

Jianan Li

Jiangsu Province Hospital

Kang Liu

Zhongshan City People's Hospital

Ryota Kobayashi

Chugai Pharmaceutical Co Ltd https://orcid.org/0000-0002-5204-2064

\section{Yuya Matsuda}

Chugai Pharmaceutical Co Ltd

\section{Zongke Zhou}

West China Hospital of Medicine: Sichuan University West China Hospital

Xisheng Weng ( $\sim$ drwengxsh@163.com )

Peking Union Medical College Hospital https://orcid.org/0000-0001-7045-230X

\section{Research article}

Keywords: NRD101, SUVENYL, Hyaluronic acid, Knee osteoarthritis, Knee pain, Knee function

Posted Date: May 19th, 2021

DOl: https://doi.org/10.21203/rs.3.rs-515957/v1

License: (c) (i) This work is licensed under a Creative Commons Attribution 4.0 International License.

Read Full License 


\section{Abstract}

\section{Background}

Intra-articular injection of hyaluronic acid (HA) has favorable effects on pain relief and knee function along with the low incidence of serious adverse reactions. Although various HA products are available for the treatment of knee osteoarthritis, it is still controversial whether differences in HA products have any clinically significant difference in efficacy and safety. In light of the above, the efficacy and safety of intra-articular injection of biological fermentation-derived high-molecular-weight HA (NRD101) was investigated in a double-blind comparative manner in Chinese patients with knee osteoarthritis.

Method

A multicenter, prospective, randomized, double-blind, parallel, active control, non-inferiority study was conducted in Chinese patients with knee osteoarthritis. Patients were randomized to receive five consecutive weekly injections of either NRD101 or Artz (a non-crosslinked low-molecular-weight HA derived from combs of roosters) followed by 4 weeks follow-up. The primary efficacy endpoint was the change from baseline in the Western Ontario and McMaster Universities Osteoarthritis Index (WOMAC) NRS pain subscale score at Week 5. Secondary efficacy endpoints included WOMAC stiffness and physical function subscale score, local pain, range of joint motion, and overall improvement.

Results

Among 267 randomized patients, 259 patients completed the study. The change from baseline in WOMAC NRS pain subscale score at Week 5 was $-2.98 \pm 0.193$ in the NRD101 group and $-2.66 \pm 0.194$ in the Artz group, and the trial met the non-inferiority criteria. Efficacy in the NRD101 group tended to be consistently higher than in the Artz group for most items of WOMAC. Several subgroup analyses also showed differences between the two groups, tending to favor NRD101. Adverse events were seen in $26.0 \%(34 / 131)$ of patients in the NRD101 group and 38.3\% (51/133) in the Artz group.

\section{Conclusions}

NRD101 improved knee pain in Chinese patients with osteoarthritis after five consecutive weekly injections. NRD101 tended to be somewhat more effective than Artz. No new safety concerns were identified.

Trial registration: JapicCTI, JapicCTI-173531. Registered 10 March 2017, https://www.clinicaltrials.jp/ctiuser/trial/Search.jsp

\section{Introduction}

Knee osteoarthritis is a degenerative disease of the knee joint involving progressive damage to the cartilage and joint against the background of genetic alterations, menopause-related estrogen deficiency, 
and aging (1). Pain in the damaged joints can severely interfere with activities of daily living. Knee osteoarthritis is one of the leading causes for reduced quality of life (2). In countries with aging populations, the numbers of patients with knee osteoarthritis are increasing (3). It has also been shown that older Chinese, especially women, have a higher prevalence of knee osteoarthritis than does the US white population (4). Knee osteoarthritis is becoming a major public health problem in China due mainly to the rapidly aging population(5).

Hyaluronic acid ( $\mathrm{HA})$ is commonly regarded as one of the most important components of the synovial fluid, and the concentration and the molecular weight of hyaluronic acid in the synovial fluid decrease as osteoarthritis progresses (6). The main purpose of intra-articular HA injections (IA-HA) is to restore the elasticity and viscosity of the synovial fluid to protect against the degradation of cartilage. The modality of IA-HA has received favorable clinical attention because of its beneficial effects on pain relief and knee function and the low incidence of serious adverse reactions $(7,8)$.

Various HA products are now available for clinical use, each with differing molecular weights (ranging from 500 to $6000 \mathrm{kDa}$ ), molecular structure (crosslinked or non-crosslinked), process of manufacturing (animal-derived or biological fermentation-derived), and so on (9). NRD101 (marketed under the brand name of SUVENYL ${ }^{\circledR}$ ) is a high-molecular-weight (HMW) HA product (average 1500-3900 kDa) produced by a biological fermentation process. NRD101 was confirmed by randomized controlled trial (RCT) to have greater effectiveness in the treatment of knee osteoarthritis in Japanese patients compared with an avian-derived low-molecular-weight (LMW) HA product (averaging 500-1200 kDa) (10). Although there are conflicting reports as to whether differences in HA products have any clinically significant difference in efficacy and safety (11), several RCTs have shown that LMW HA products of $800-1500 \mathrm{kDa}$ are superior to other HA products of lower molecular weight (500-730 kDa) in efficacy with a similar safety profile (12) and that biological fermentation-derived HMW HA (2400-3600 kDa) has a better safety profile than that of avian-derived crosslinked HMW HA (6000 kDa) with comparable efficacy (13).

In light of the above, we conducted a multicenter, prospective, randomized, double-blind, positive-drug, parallel, active control, non-inferiority study to compare the efficacy and safety of IA injection of biological fermentation-derived HMW HA (NRD101) with that of avian-derived LMW HA (Artz) in Chinese patients with knee osteoarthritis.

\section{Methods}

\section{Study design}

This confirmatory study was carried out from July 2017 to July 2018 as a multicenter, prospective, randomized, double-blind, parallel, active control, non-inferiority study.

Patients were randomized in a 1:1 ratio by a computerized system to receive either a biological fermentation-derived HMW HA product, named NRD101/SUVENYL ${ }^{\circledR}$ (Chugai Pharmaceutical Co., Ltd., Tokyo, Japan) with an average molecular weight of 1500-3900 kDa or a non-crosslinked LMW HA 
product derived from roosters' combs, named Artz/ARTZ ${ }^{\circledR}$ (also marketed under the names of Artzal or Supartz) (Seikagaku Corporation, Tokyo, Japan) with an average molecular weight of 500-1200 kDa. Patients received $2.5 \mathrm{~mL}$ injections of the assigned study drug (NRD101 or Artz) into the knee joint cavity once a week for 4 consecutive weeks (a total of five injections from Week 0 to Week 5). Needle puncture and aspiration were conducted where appropriate. The use of local anesthetics was prohibited. If the subject had osteoarthritis in both knees, only one knee (not both) was treated. A standard manual and necessary training for the injection method was provided to investigators before study initiation. Evaluation of the primary endpoint was carried out 1 week after the final dose (at Week 5). Final evaluation of efficacy and safety was carried out 4 weeks after the final dose (at Week 8). To ensure study blindness, the investigator who administered the study drug was not allowed to evaluate the patients he or she had treated.

The study was registered with JapicCTI (Japic ID: JapicCTI-173531).

\section{Patients}

Ambulatory outpatients aged 40 to less than 80 meeting the following criteria were enrolled: diagnosed with knee osteoarthritis based on the American College of Rheumatology (ACR) clinical classification criteria; classified as Kellgren-Lawrence (K-L) Grade 2 or Grade 3 at the tibiofemoral joint of the study knee, with the grade of the contralateral knee not exceeding that of the study knee; chronic pain lasting for at least 1 month prior to the signature date of the informed consent form; and a mean Western Ontario and McMaster Universities Osteoarthritis Index (WOMAC) for pain (five items) determined at Week 0 of at least 5 on an 11-point numeric rating scale (NRS). Patients diagnosed with secondary knee osteoarthritis or other diseases/disorders causing functional impairment of the knee were excluded from this study. Patients who had rheumatic diseases, knee osteoarthritis on the contralateral knee requiring medical treatment into the knee joint cavity, or a history of lower-extremity arthroplasty were also excluded. Skin diseases or infections around the area of injection site, serious cardiac disorders, renal failure, hematologic diseases, diabetes mellitus, and severe hepatic impairment were set as exclusion criteria for safety reasons. The following medications or therapies were prohibited during the following defined periods up to the end-of-study visit: opioids for knee osteoarthritis before and during the study, local injections of HA into either knee, or opioids for pain other than for knee osteoarthritis from 24 weeks before the first study dose; local injection of corticosteroids or anesthetics into either knee, external application of corticosteroids for either knee, oral, suppository, or intravenous doses of corticosteroids from 4 weeks before the first study; and surgical treatment of either knee, exercise therapy for either knee, or Chinese patent medicines used to treat knee osteoarthritis from 2 weeks before the first study dose. External application of anti-inflammatory or analgesic medication to the study knee, oral, suppository, or intravenous administration of anti-inflammatory or analgesic medication, physical or orthotic therapy of the knee, muscle relaxants, and psychotropic agents (including somnifacients) were not allowed to be newly started and if the patient was already receiving any of them at the start of the period, the treatment regimens were not allowed to be changed during the study. Acetaminophen up to $4 \mathrm{~g}$ a day was allowed for reducing the pain as a rescue therapy during the study. 


\section{Outcomes}

The demographics and baseline characteristics of patients were collected. The following clinical outcomes were assessed and reported: WOMAC Osteoarthritis Index NRS 3.1 simplified Chinese for mainland China on an 11-point Numerical Rating Scale (0 to 10) consisting of pain, stiffness, and physical function domains; local pain consisting of spontaneous pain, tenderness, nighttime pain, and movement pain scored on a 4-item scale ( 0 , none; 1 , mild; 2 , moderate; 3 , severe); range of motion in the joint (angles of extension and flexion of the knee joint); and overall improvement ( 3 to +3 from baseline). WOMAC was a patient-reported outcome and the other outcomes were assessed by investigators. The primary efficacy endpoint was the change from baseline in WOMAC NRS pain at Week 5. Secondary efficacy endpoints included WOMAC stiffness and physical function, local pain, range of motion in the joint, and overall improvement.

All adverse events (AEs) reported by patients were also recorded at each visit, and clinical laboratory examinations for hematology and serum chemistry were conducted at screening, Week 5 (or withdrawal), and Week 8.

\section{Statistical analysis}

All randomized patients who received at least one dose of the study drug were included in the safety analysis set, and all randomized patients who received at least one dose of the study drug and for whom WOMAC NRS pain data were available for baseline and one or more time points after the start of the study treatment were included in the full analysis set. The full analysis set was the target population for primary efficacy analysis.

Based on the meta-analysis conducted as part of the Osteoarthritis Research Society International (OARSI) recommendations, the effect sizes of hyaluronic acid preparations for intra-articular injection treatment of knee osteoarthritis were calculated to be 0.60 (95\% confidence interval $[\mathrm{Cl}] 0.37,0.83)(14)$. The same effect sizes (0.60) were assumed for improvements in the WOMAC Visual Analogue Scale (VAS) pain score by treatment with NRD101 and Artz. In addition, the standard deviations for the changes in WOMAC VAS pain were assumed to be $2.5 \mathrm{~cm}$ for NRD101 and Artz therapies. Using these assumptions, the lower limit of the two-sided $95 \% \mathrm{Cl}$ for the difference in WOMAC VAS pain (Artz placebo) was computed to be $-0.925 \mathrm{~cm}$. The score will be extrapolated as -0.925 points in the case of using the 11-point NRS. Consequently, the non-inferiority margin was set at -0.925 points in this study. To demonstrate the non-inferiority of NRD101 versus Artz with a power of $80 \%$, a total of 232 patients in two arms of 116 was necessary. The target population size was 260 patients (two arms of 130 each) taking into consideration drop-outs and other factors reducing the sample size.

Subject demographics and baseline data (continuous and categorical) were presented for both treatment arms using summary statistics. 
For the primary endpoint, the non-inferiority of NRD101 versus Artz was investigated for the change from baseline of WOMAC NRS pain at Week 5. The least-squares estimate of two-sided $95 \% \mathrm{Cl}$ for the difference (NRD101 - Artz) in the primary endpoint was determined using an analysis of covariance (ANCOVA) model with baseline WOMAC NRS pain and K-L Grade as covariates. The criterion for noninferiority would be met unless the lower limit of the $\mathrm{Cl}$ was below the non-inferiority margin of -0.925 points. Secondary analyses focused on data summaries (i.e., descriptive statistics) of the primary and secondary endpoints in the full analysis set and the per-protocol set by planned treatment arm. The summary of WOMAC NRS included the rating for each question, the subscale scores of the three subsections, and the change from baseline at each visit. Transitions in the subscale scores of WOMAC questions were plotted as appropriate.

AEs were codified according to MedDRA (version 20.0), and the numbers of subjects who experienced AEs as well as the numbers of AEs themselves (i.e., incidence) were tabulated by event according to MedDRA preferred term. AEs for which a causal relationship with the study drug could not be ruled out were classified as adverse reactions to the study drug and, where necessary, tabulated separately to other AEs according to the number of subjects and incidence. The severity of AEs was assessed according to the following criteria (mild: discomfort noticed, but no disruption of normal daily activity; moderate: discomfort sufficient to reduce or affect normal daily activity; severe: incapacitating with inability to work or to perform normal daily activity). AEs that met any of the following criteria were handled as serious AEs: is fatal, is life threatening, requires or prolongs inpatient hospitalization, results in persistent or significant disability/incapacity, results in congenital anomaly/birth defect in a neonate/infant born to a mother exposed to the investigational product, or is a significant medical event in the investigator's judgment. The safety analysis set was employed for safety analysis.

Statistical analyses including subgroup analyses were pre-specified in the statistical analysis plan before the database was locked and the randomization codes opened.

Randomization was conducted according to two stratification factors consisting of WOMAC NRS pain (no more than 7 or greater than 7 points) and K-L Grade (2 or 3).

\section{Results}

Patient disposition and demographics

A total of 351 patients were screened from 14 sites. Of these, 84 failed screening, and finally 267 patients were randomized, including 133 in the NRD101 group (test group) and 134 in the Artz group (control group). Among all randomized patients, 259 patients (97\%) completed the study and 8 patients (3\%) discontinued the study (Fig. 1). The reasons for early discontinuation were as follows: five patients withdrew consent, including two from the NRD101 group and three from the Artz group; two patients discontinued due to the occurrence of an AE, all in the Artz group; one patient in the NRD101 group was withdrawn due to being mistakenly injected with Artz (this discontinuation was at the suggestion of the 
site's ethics committee). In whole, the persistence rate of subjects in this study remained high, and there was no clear difference in early discontinuation between the two groups.

For analysis set classification, 264 subjects were included in the safety analysis set, including 131 in the NRD101 group and 133 in the Artz group; 250 subjects were included in the full analysis set, including 127 in the NRD101 group and 123 in the Artz group; 230 subjects were included in the per protocol set, including 118 in the NRD101 group and 112 in the Artz group. Three subjects were excluded from the safety analysis set due to a violation of the informed consent process (an unblinded investigator signed the informed consent form as an impartial witness of these illiterate subjects).

Of the 250 patients in the full analysis set, $82.0 \%$ were female, the mean age was $60.9 \pm 8.19$ years old, and the mean body mass index was $25.3 \pm 3.39 \mathrm{~kg} / \mathrm{m}^{2}$. The mean baseline of WOMAC NRS pain was $6.60 \pm 1.135$, and the median duration of chronic pain of study knee was 1.88 years. For K-L grade, $48.8 \%$ of subjects were judged as Grade 2. There was no difference in baseline characteristics between the two groups (Table 1).

\section{Efficacy outcomes}

In the full analysis set, the change in WOMAC NRS pain from baseline on a Last Observation Carried Forward basis at Week 5 was $-2.98 \pm 0.193$ points in the NRD101 group and $-2.66 \pm 0.194$ points in the Artz group; the difference between the two groups was $0.33 \pm 0.258$ points with a $95 \% \mathrm{Cl}$ of -0.18 to 0.83 points. The lower limit of $95 \% \mathrm{Cl}$ was -0.18 points, which was not below the non-inferiority margin of -0.925 points. Therefore, it is considered that the NRD101 group was not inferior to the Artz group, and the trial attained the primary efficacy end point. The improvement in WOMAC pain in the NRD101 group tended to be greater than in the Artz group from Week 3 to the end of the study (Fig. 2). The change in WOMAC NRS stiffness from baseline at Week 5 was $-2.63 \pm 2.393$ points in the NRD101 group and -2.21 \pm 2.422 points in the Artz group, and the change in WOMAC NRS physical function was $-2.48 \pm 2.155$ in the NRD101 group and $-2.31 \pm 1.986$ in the Artz group. For most items of the WOMAC, the NRD101 group consistently tended to show greater improvement from baseline at Week 5 than did the Artz group (Table 2). On the other hand, rates of improvement of local pain were similar between the NRD101 and Artz groups (Table 3). The range of motion in the joint was significantly improved compared with baseline in both treatment groups although no difference between the groups was observed. The mean change in maximum extension angle (degree) from baseline at Week 5 was $-1.13(95 \% \mathrm{Cl}-1.70,-0.55)$ in the NRD101 group and $-1.22(95 \% \mathrm{Cl}-1.76,-0.68)$ in the Artz group; the mean change in maximum flexion angle (degree) from baseline at Week 5 was $7.48(95 \% \mathrm{Cl} 5.66,9.30)$ in the NRD101 group and 5.93 $(95 \% \mathrm{Cl} 4.13,7.72)$ in the Artz group. The number of patients showing an overall improvement ([number showing $\leq 2$-point change from baseline]/[total number of patients]) at Week 5 was higher in the NRD101 group (60.0\%) than in the Artz group (53.4\%), but no statistically significant difference was observed between the two groups (odds ratio: 1.31, [95\% $\mathrm{Cl} 0.79,2.18]$ ). 
The results of the pre-specified subgroup analyses of primary outcome (WOMAC NRS pain change at Week 5) were essentially consistent with the results in the overall population, with NRD101 tending to be more effective than Artz in most subgroups (Table 4). Among them, NRD101 showed the tendency of greater pain reduction than Artz in the subgroup of patients whose duration of chronic pain was $>1$ year and $\leq 5$ years (difference: $-1.01[95 \% \mathrm{Cl}-1.83,-0.19]$ ) and patients whose body weight was $>50 \mathrm{~kg}$ and $\leq 60 \mathrm{~kg}$ (difference: $-1.22[95 \% \mathrm{Cl}-2.23,-0.21])$.

Overall, there was no difference in the use of acetaminophen as a rescue therapy between the two groups.

Safety

Among all subjects in the safety analysis set, the percentage of subjects with AEs was $32.2 \%(85 / 264)$ : $26.0 \%(34 / 131)$ in the NRD101 group and 38.3\% (51/133) in the Artz group (Table 5). The most common AEs were viral upper respiratory tract infection 5.3\% (14/264), upper respiratory tract infection $3.8 \%$ (10/264), and arthralgia 3.0\% (8/264). There was no difference between the two groups in the incidence of arthralgia (3.1\% [4/131] for NRD101 and 3.0\% [4/133] for Artz). As for injection site-related events, there was only one subject with injection site erythema and one subject with injection site pain in the NRD101 group, and one subject with injection site rash in the Artz group. The proportion of subjects who experienced severe AEs was $0.8 \%$ in the NRD101 group and $2.3 \%$ in the Artz group. Two out of 264 subjects (0.8\%) developed serious AEs: sleep apnea syndrome (one event in one subject in the NRD101 group) and hypertension worsened (one event in one subject in the Artz group). Neither serious AE was related to the study drug, and the dose of study drug remained unchanged. Among all subjects in the safety analysis set, $14(5.3 \%)$ experienced 20 adverse reactions: $4.6 \%(6 / 131)$ in the NRD101 group and $6.0 \%(8 / 133)$ in the Artz group. AEs leading to discontinuation occurred only in the Artz group (one subject due to arthralgia and plica syndrome, one subject due to rash). No subject died during the study. Thus, no new safety concerns were identified throughout the study.

\section{Discussion}

In this study, we evaluated the efficacy and safety of five consecutive intra-articular injections of HMW HA derived from a biological fermentation process (NRD101) into patients with osteoarthritis of the knee, and we succeeded in verifying its non-inferiority to avian-derived LMW HA (Artz) in the symptomatic improvement of pain. In addition, for many efficacy endpoints (e.g., treatment effect in each domain of WOMAC, overall improvement, etc.), NRD101 tended to be consistently more effective than Artz. Furthermore, statistically significant differences were observed in some subgroup analyses of WOMAC pain reduction, while the opposite result (the effect of Artz being significantly greater than NRD101) was not observed in any of the pre-defined subgroup analyses, suggesting that HMW HA may be more powerful than LMW HA in terms of control of clinical symptoms. These findings are essentially consistent with the results of previous RCTs in which NRD101 or intermediate-molecular-weight HA (GOON; Rottapharm Madaus, Monza, Italy) were found to have better efficacy than LMW HA $(10,12)$. A head- 
to-head RCT designed to confirm superiority is considered necessary to conclude whether the differences in efficacy observed here are truly clinically meaningful or not.

In terms of safety, there were trends toward fewer AEs in the NRD101 group than in the Artz group, and the numbers of severe AEs, adverse reactions to the HA preparation, and AEs leading to trial discontinuation also tended to be less in the NRD101 group than in the Artz group. These two formulations have different manufacturing processes: NRD101 is a HA derived from a biological fermentation process, and Artz is avian-derived HA. These differences might influence safety outcomes. A systematic review that aimed to determine product differences in HAs for osteoarthritis of the knee showed that biological fermentation-derived HAs caused a significantly smaller incidence of effusion compared with avian-derived HAs, and also the former demonstrated fewer acute flare-ups at the injection site than did the latter (15). Nevertheless, in the current study, the incidence rates of AEs of interest, such as injection site-related events and arthralgia were not different between the two groups; therefore, it is impossible to rule out the possibility that the differences in incidence rates of AEs observed in this study were a chance result. In addition, in recent years, potential safety concerns have arisen about whether crosslinked HMW HA may pose a higher risk of injection site-related events such as painful acute local reactions (16). Thus, further investigations including real-world data are required in order to clarify the differences in safety profiles among different HA formulations.

NRD101 has the intrinsic properties of endogenous HA, especially in terms of molecular weight and production process. The molecular weight of NRD101 is comparatively high but is regarded as close to that of endogenous HA in healthy knee joints (17). In addition, NRD101 is produced by a biological fermentation process (and is not animal-derived), without artificial cross-linking. The results of this study and accumulated evidence suggest that NRD101 may provide a better effect with respect to symptomatic pain and function with fewer AEs compared with animal-derived LMW HAs, and may also have a better safety profile than that of artificially crosslinked HMW HA products.

However, this study has a number of several limitations. First, this study was not a placebo-controlled study. In trials of knee osteoarthritis, intra-articular saline injections are usually employed as the "placebo" control (IA-placebo). In some previous trials examining the treatment effect of IA-HA compared with IAplacebo, results obtained were not consistent, probably due to the lack of unity of the trial designs (18, 19), and there is still uncertainty with regard to the therapeutic effects of different types of HA products on knee osteoarthritis (16). Therefore, in this study, we carefully considered which comparator drug to use and we selected Artz, which has robust evidence showing efficacy and safety superior to that of IAplacebo. For instance, its superiority over IA-placebo has been strongly confirmed by an individual-patientdata-driven meta-analysis selecting only clinical trials that used Artz (20). Although our study results combined with the evidence of Artz suggest that NRD101 would be superior to IA-placebo in symptomatic endpoints, there is no doubt that direct comparison with IA-placebo would provide more robust evidence. Second, the planned observations were of relatively short-term clinical symptoms and functional improvement. In other words, the study period was just 8 weeks including 4 weeks after the end of the last dose, and it was not designed to observe long-term effects. Although the carryover effect of repeated 
IA-HA has been reported for other HA products (21), it is not realistic to extrapolate that data to NRD101 directly. Third, the effect on cartilage preservation was not assessed in this study. Recent clinical evidence in patients with knee osteoarthritis has shown that IA-HA extends the period before total knee replacement surgery is required (22) and preserves knee-joint cartilage (23). NRD101 showed a preventive effect on cartilage destruction in a rabbit model of knee osteoarthritis induced by partial meniscectomy (24), but its actual effect on humans is still unclear. Altman et al. (17) suggested on the basis of basic research that a higher molecular weight HA product might provide better chondroprotective effects.

Disease modification properties including preservation of cartilage are clinically important and need to be investigated through RCTs with different HA products.

\section{Conclusions}

In conclusion, NRD101 as a biological fermentation-derived HMW HA product improved knee pain in patients with osteoarthritis after five consecutive weekly injections, and was non-inferior to Artz. In addition, NRD101 tended to be consistently more effective than Artz in most items of WOMAC, and several subgroup analyses showed significant differences between the two groups, favoring NRD101. No new safety concerns were identified. On the other hand, it is not possible to conclude whether the differences seen here between the two HA products truly contribute to improved clinical benefit, necessitating a superiority trial on a larger scale and with a longer follow-up period.

\section{Abbreviations}

ACR: American College of Rheumatology; AE: Adverse event; Cl: Confidence interval; HA: Hyaluronic acid; HMW: High-molecular-weight; IA-HA: Intra-articular HA injections; K-L: Kellgren-Lawrence; LMW: Lowmolecular-weight; NRS: Numeric rating scale; OARSI: Osteoarthritis Research Society International; RCT: Randomized controlled trial; VAS: Visual Analogue Scale; WOMAC: Western Ontario and McMaster Universities Osteoarthritis Index

\section{Declarations}

\section{Funding}

This study was funded by Chugai Pharmaceutical Co., Ltd. The sponsor planned this study and implemented data collection, analysis, and interpretation in conjunction with the lead principal investigator and scientific advisor. The sponsor also provided editorial and writing support for this manuscript.

\section{Ethics approval and consent to participate}

This study was conducted in accordance with the principles laid down by the Declaration of Helsinki (Edinburgh, 2008), and was also conducted in compliance with Good Clinical Practice (GCP) and the national laws and regulations of China. The study conduct was approved by the China Food and Drug 
Administration (Approval No. 2016L08917). The protocol was approved by the ethics committee at each center, and informed consent was obtained from each patient.

\section{Consent for publication}

Not applicable

\section{Acknowledgements}

The following investigators participated in this trial: Qingyun Xue, Beijing Hospital; Qing He, Navy General Hospital PLA China; Jingmin Huang, Tianjin Hospital; Biao Cheng, Shanghai Tenth People's Hospital; Liaobin Chen, Zhongnan Hospital of Wuhan University; Zhanjun Shi, Nanfang Hospital, Southern Medical University; Lijun Liu, Shenzhen Second People's Hospital; Yaping Li, General Hospital of Ningxia Medical University; Shijun Gao, The Third Hospital of Hebei Medical University. R\&G PharmaStudies Co., Ltd. (Beijing, China) also supported this study as a contract research organization.

\section{Authors' contributions}

Haoyang Wang and Wei Wang participated in interpretation of the study data and drafted this article. Zongke Zhou and Xisheng Weng contributed to study planning and design, and critically revised the manuscript for important intellectual content. Ryota Kobayashi supported the implementation of this study and provided administrative, technical, and logistic support. Yuya Matsuda provided statistical expertise and led data analysis and interpretation. Yongsheng $\mathrm{Xu}$, Jianan $\mathrm{Li}$, and Kang Liu contributed to patient enrolment for the study. All authors have made substantial contributions to the review and final approval of this manuscript.

\section{Competing interests}

$H W, W W, Y X, J L, K L, Z Z$ and $X W e n g$ declare that they have no conflict of interest. RK and YM are employees of Chugai Pharmaceutical Co., Ltd.

\section{Availability of data and materials}

Qualified researchers may request access to individual patient level data through the clinical study data request platform (www.clinicalstudydatarequest.com). For further details on Chugai's Data Sharing Policy and how to request access to related clinical study documents, see www.chugaipharm.co.jp/english/profile/rd/ctds_request.html.

\section{References}

1. Herrero-Beaumont G, Roman-Blas JA, Castaneda S, Jimenez SA. Primary osteoarthritis no longer primary: three subsets with distinct etiological, clinical, and therapeutic characteristics. Seminars in arthritis and rheumatism. 2009;39(2):71-80. 
2. Vitaloni M, Botto-van Bemden A, Sciortino Contreras RM, Scotton D, Bibas M, Quintero M, et al. Global management of patients with knee osteoarthritis begins with quality of life assessment: a systematic review. BMC Musculoskelet Disord. 2019;20(1):493.

3. Shane Anderson A, Loeser RF. Why is osteoarthritis an age-related disease? Best practice \& research Clinical rheumatology. 2010;24(1):15-26.

4. Zhang Y, Xu L, Nevitt MC, Aliabadi P, Yu W, Qin M, et al. Comparison of the prevalence of knee osteoarthritis between the elderly Chinese population in Beijing and whites in the United States: The Beijing Osteoarthritis Study. Arthritis Rheum. 2001;44(9):2065-71.

5. Peng X. China's demographic history and future challenges. Science (New York, NY). 2011;333(6042):581-7.

6. Fam H, Bryant JT, Kontopoulou M. Rheological properties of synovial fluids. Biorheology. 2007;44(2):59-74.

7. Cooper C, Rannou F, Richette P, Bruyere O, Al-Daghri N, Altman RD, et al. Use of Intraarticular Hyaluronic Acid in the Management of Knee Osteoarthritis in Clinical Practice. Arthritis Care Res (Hoboken). 2017;69(9):1287-96.

8. Ishijima M, Nakamura T, Shimizu K, Hayashi K, Kikuchi H, Soen S, et al. Intra-articular hyaluronic acid injection versus oral non-steroidal anti-inflammatory drug for the treatment of knee osteoarthritis: a multi-center, randomized, open-label, non-inferiority trial. Arthritis Res Ther. 2014;16(1):R18.

9. Bowman S, Awad ME, Hamrick MW, Hunter M, Fulzele S. Recent advances in hyaluronic acid based therapy for osteoarthritis. Clinical and translational medicine. 2018;7(1):6.

10. Yamamoto M, Sugarawa S, Tsukamoto Y, Motegi M, Iwata H, Ryu J. Clinical evaluation of high molecular weight sodium hyaluronate (NRD101) on osteoarthritis of the knee. A phase III comparative clinical study with Artz as a control drug. Jpn Pharmacol Ther. 1994;22(9):4059-87.

11. Reichenbach S, Blank S, Rutjes AW, Shang A, King EA, Dieppe PA, et al. Hylan versus hyaluronic acid for osteoarthritis of the knee: a systematic review and meta-analysis. Arthritis Rheum. 2007;57(8):1410-8.

12. Berenbaum F, Grifka J, Cazzaniga S, D'Amato M, Giacovelli G, Chevalier X, et al. A randomised, double-blind, controlled trial comparing two intra-articular hyaluronic acid preparations differing by their molecular weight in symptomatic knee osteoarthritis. Ann Rheum Dis. 2012;71(9):1454-60.

13. Kirchner M, Marshall D. A double-blind randomized controlled trial comparing alternate forms of high molecular weight hyaluronan for the treatment of osteoarthritis of the knee. Osteoarthritis and cartilage. 2006;14(2):154-62.

14. Zhang W, Nuki G, Moskowitz RW, Abramson S, Altman RD, Arden NK, et al. OARSI recommendations for the management of hip and knee osteoarthritis: part III: Changes in evidence following systematic cumulative update of research published through January 2009. Osteoarthritis and cartilage. 2010;18(4):476-99.

15. Altman RD, Bedi A, Karlsson J, Sancheti P, Schemitsch E. Product Differences in Intra-articular Hyaluronic Acids for Osteoarthritis of the Knee. The American journal of sports medicine. 
2016;44(8):2158-65.

16. Wang CT, Lin J, Chang CJ, Lin YT, Hou SM. Therapeutic effects of hyaluronic acid on osteoarthritis of the knee. A meta-analysis of randomized controlled trials. The Journal of bone and joint surgery American volume. 2004;86(3):538-45.

17. Altman RD, Manjoo A, Fierlinger A, Niazi F, Nicholls M. The mechanism of action for hyaluronic acid treatment in the osteoarthritic knee: a systematic review. BMC Musculoskelet Disord. 2015;16:321.

18. Karlsson J, Sjogren LS, Lohmander LS. Comparison of two hyaluronan drugs and placebo in patients with knee osteoarthritis. A controlled, randomized, double-blind, parallel-design multicentre study. Rheumatology (Oxford, England). 2002;41(11):1240-8.

19. Johansen $M$, Bahrt $H$, Altman RD, Bartels EM, Juhl CB, Bliddal $H$, et al. Exploring reasons for the observed inconsistent trial reports on intra-articular injections with hyaluronic acid in the treatment of osteoarthritis: Meta-regression analyses of randomized trials. Seminars in arthritis and rheumatism. 2016;46(1):34-48.

20. Strand V, Conaghan PG, Lohmander LS, Koutsoukos AD, Hurley FL, Bird H, et al. An integrated analysis of five double-blind, randomized controlled trials evaluating the safety and efficacy of a hyaluronan product for intra-articular injection in osteoarthritis of the knee. Osteoarthritis and cartilage. 2006;14(9):859-66.

21. Navarro-Sarabia F, Coronel P, Collantes E, Navarro FJ, de la Serna AR, Naranjo A, et al. A 40-month multicentre, randomised placebo-controlled study to assess the efficacy and carry-over effect of repeated intra-articular injections of hyaluronic acid in knee osteoarthritis: the AMELIA project. Ann Rheum Dis. 2011;70(11):1957-62.

22. Altman R, Lim S, Steen RG, Dasa V. Hyaluronic Acid Injections Are Associated with Delay of Total Knee Replacement Surgery in Patients with Knee Osteoarthritis: Evidence from a Large U.S. Health Claims Database. PLoS One. 2015;10(12):e0145776.

23. Wang Y, Hall S, Hanna F, Wluka AE, Grant G, Marks P, et al. Effects of Hylan G-F 20 supplementation on cartilage preservation detected by magnetic resonance imaging in osteoarthritis of the knee: a two-year single-blind clinical trial. BMC Musculoskelet Disord. 2011;12:195.

24. Mihara M, Higo S, Uchiyama Y, Tanabe K, Saito K. Different effects of high molecular weight sodium hyaluronate and NSAID on the progression of the cartilage degeneration in rabbit OA model. Osteoarthritis and cartilage. 2007;15(5):543-9.

\section{Tables}

Table 1. Baseline demographics (full analysis set) 


\begin{tabular}{|c|c|c|}
\hline & NRD101 (N=127) & Artz $(\mathrm{N}=123)$ \\
\hline Female, n (\%) & $104(81.9 \%)$ & $101(82.1 \%)$ \\
\hline Mean age, years (SD) & $61.1(8.59)$ & $60.7(7.78)$ \\
\hline Mean height, cm (SD) & $161.70(7.024)$ & $161.50(7.189)$ \\
\hline Mean body weight, $\mathrm{kg}$ (SD) & $66.29(10.286)$ & $66.03(10.576)$ \\
\hline Mean BMI, kg/m² (SD) & $25.30(3.244)$ & $25.30(3.547)$ \\
\hline \multicolumn{3}{|c|}{ Baseline WOMAC NRS pain, score $(0-10)$} \\
\hline Mean (SD) & $6.58(1.140)$ & $6.62(1.133)$ \\
\hline Median & 6.40 & 6.40 \\
\hline \multicolumn{3}{|c|}{ Duration of chronic pain in study knee, years } \\
\hline Mean (SD) & $3.23(4.012)$ & $3.75(4.685)$ \\
\hline Median & 1.53 & 2.27 \\
\hline \multicolumn{3}{|c|}{ Category of duration of chronic pain, $\mathrm{n}(\%)$} \\
\hline$\leq 6$ months & $40(31.5 \%)$ & $29(23.6 \%)$ \\
\hline$>6$ months $-\leq 1$ year & $12(9.4 \%)$ & $15(12.2 \%)$ \\
\hline$>1$ year $-\leq 5$ years & $46(36.2 \%)$ & $51(41.5 \%)$ \\
\hline$>5$ years & $29(22.8 \%)$ & $28(22.8 \%)$ \\
\hline \multicolumn{3}{|l|}{ Study knee } \\
\hline Right knee, n (\%) & $74(58.3 \%)$ & $61(49.6 \%)$ \\
\hline \multicolumn{3}{|c|}{ KL grade of tibiofemoral joint of study knee } \\
\hline 2 (minimal) & $62(48.8 \%)$ & $60(48.8 \%)$ \\
\hline 3 (moderate) & 65 (51.2\%) & 63 (51.2\%) \\
\hline
\end{tabular}

$\mathrm{SD}=$ standard deviation; $\mathrm{BMI}=$ body mass index; $\mathrm{NRS}=$ numeric rating scale; $\mathrm{KL}=$ Kellgren - Lawrence Table 2. Forest plot of changes from baseline for each item of WOMAC at Week 5 (full analysis set) 

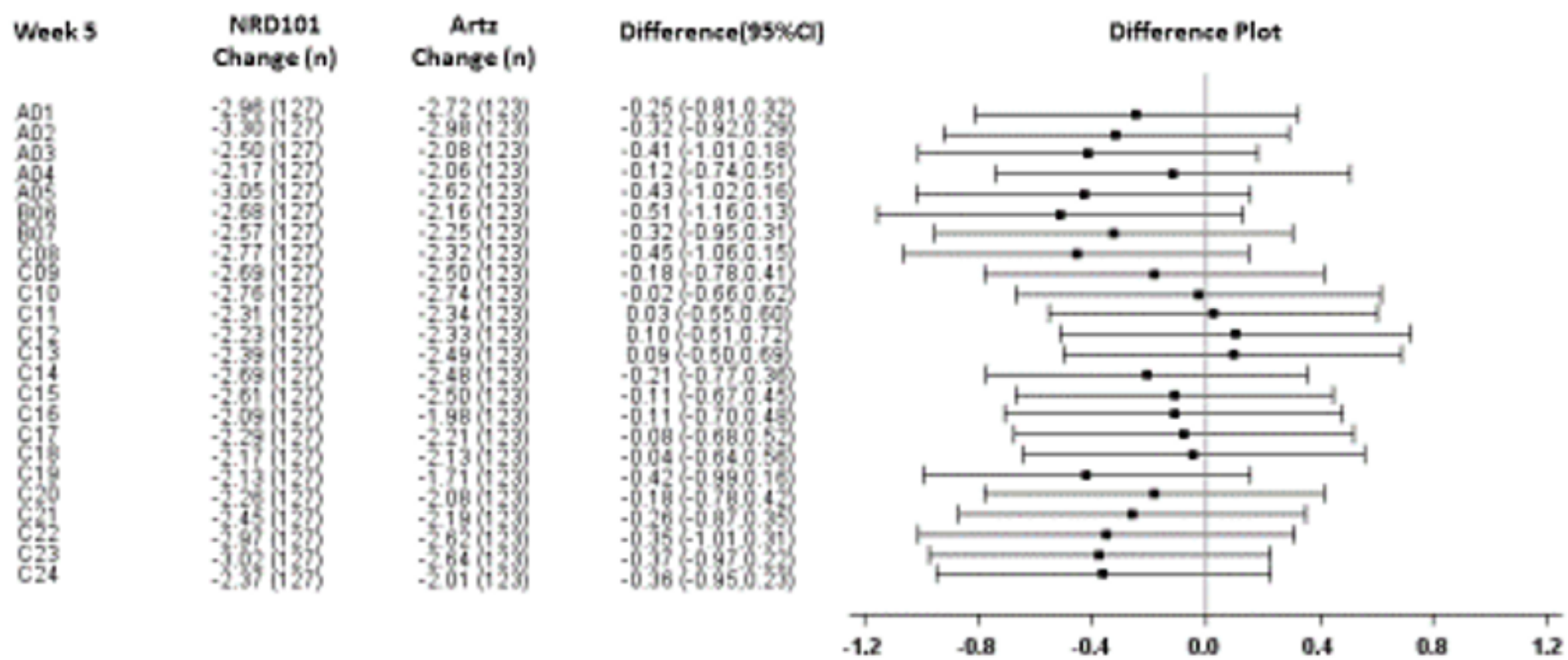

NRD101 Better

Artz Better

Categories of WOMAC (24 items):

Pain (5 items): walking on flat surface (A01), going up or down stairs (A02), at night while in bed (A03), sitting or lying (A04), and standing upright (A05)

Stiffness (2 items): after first awakening in the morning (B06) and after periods of inactivity later in the day (B07)

Physical function (17 items): descending stairs (C08), ascending stairs (C09), getting out of a chair (C10), remaining in a standing position (C11), bending to pick something up off the floor (C12), walking on a flat surface (C13), getting in and out of a car (C14), going shopping (C15), putting on socks or stockings (C16), getting out of bed (C17), taking off socks or stockings (C18), lying in bed (C19), getting in and out of the bathtub/shower (C20), remaining in a sitting position (C21), getting on or off the toilet (C22), performing heavy domestic duties (C23), and performing light domestic duties (C24)

\section{Table 3. Improvement rate for local pain* (full analysis set)}




\begin{tabular}{|c|c|c|c|c|c|c|c|c|c|}
\hline \multirow{3}{*}{ Item } & \multirow{3}{*}{ Arm } & \multicolumn{8}{|c|}{ Score difference (Week 5 - Baseline) } \\
\hline & & \multicolumn{3}{|c|}{ Improved } & \multirow{2}{*}{$\begin{array}{l}\text { No } \\
\text { change } \\
0\end{array}$} & \multicolumn{3}{|c|}{ Worsened } & \multirow{2}{*}{$\begin{array}{l}\text { Percentage of improved } \\
\text { patients (Improved/Total) }\end{array}$} \\
\hline & & -3 & -2 & -1 & & +1 & +2 & +3 & \\
\hline \multirow{2}{*}{$\begin{array}{l}\text { Spontaneous } \\
\text { pain }\end{array}$} & NRD101 & 2 & 23 & 60 & 36 & 5 & 1 & 0 & $66.9 \%(85 / 127)$ \\
\hline & Artz & 5 & 27 & 46 & 41 & 3 & 1 & 0 & $63.4 \%(78 / 123)$ \\
\hline \multirow[t]{2}{*}{ Tenderness } & NRD101 & 4 & 34 & 53 & 33 & 3 & 0 & 0 & 71.7\% (91/127) \\
\hline & Artz & 9 & 31 & 48 & 32 & 3 & 0 & 0 & $71.5 \%(88 / 123)$ \\
\hline \multirow{2}{*}{$\begin{array}{l}\text { Nighttime } \\
\text { pain }\end{array}$} & NRD101 & 2 & 25 & 56 & 42 & 1 & 1 & 0 & $65.4 \%(83 / 127)$ \\
\hline & Artz & 4 & 17 & 48 & 52 & 2 & 0 & 0 & $56.1 \%(69 / 123)$ \\
\hline \multirow{2}{*}{$\begin{array}{l}\text { Movement } \\
\text { pain }\end{array}$} & NRD101 & 2 & 36 & 62 & 25 & 2 & 0 & 0 & $78.7 \%(100 / 127)$ \\
\hline & Artz & 8 & 30 & 61 & 24 & 0 & 0 & 0 & $80.5 \%(99 / 123)$ \\
\hline
\end{tabular}

*As assessed by an investigator.

Table 4. Pre-defined subgroup analysis of primary outcome (change in WOMAC NRS pain at Week 5) (full analysis set) 


\begin{tabular}{|c|c|c|c|}
\hline & $\begin{array}{c}\text { NRD } \\
\text { Change (n) }\end{array}$ & $\begin{array}{c}\text { Artz } \\
\text { Change (n) }\end{array}$ & Diffrence(95\%Cl) \\
\hline Total Group & $-2.80(127)$ & $-2.49(123)$ & $-0.30(-0.82,0.21)$ \\
\hline \multicolumn{4}{|l|}{ Sex } \\
\hline Female & $-2.74(104)$ & $-2.44(101)$ & $-0.30(-0.86,0.27)$ \\
\hline Male & $-3.05(23)$ & $-2.73(22)$ & $-0.32(-1.62,0.97)$ \\
\hline \multicolumn{4}{|l|}{ Age } \\
\hline$<=49$ year & $-3.18(10)$ & $-3.20(8)$ & $0.02(-2.18,2.22)$ \\
\hline 50year-59year & $-2.66(45)$ & $-2.43(46)$ & $-0.23(-1.14,0.68)$ \\
\hline 60year-69year & $-3.02(50)$ & $-2.61(54)$ & $-0.40(-1.13,0.33)$ \\
\hline s=70year & $-2.39(22)$ & $-1.85(15)$ & $-0.54(-2.08,1.00)$ \\
\hline \multicolumn{4}{|l|}{ K-L Grade Score at BL } \\
\hline 2 & $-3.04(62)$ & $-2.57(64)$ & $-0.47(-1.21,0.27)$ \\
\hline 3 & $-2.56(65)$ & $-2.40(59)$ & $-0.16(-0.88,0.57)$ \\
\hline \multicolumn{4}{|c|}{ WOMAC Pain Score at BL } \\
\hline$<=7$ & $-2.58(93)$ & $-2.25(88)$ & $=0.34(-0.92,0.24)$ \\
\hline$>7$ & $-3.37(34)$ & $-3.10(35)$ & $-0.27(-1.33,0.79)$ \\
\hline \multicolumn{4}{|c|}{ Duration of Chronic Pain } \\
\hline$<=6$ months & $-2.32(40)$ & $-3.16(29)$ & $0.84(-0.18,1.85)$ \\
\hline$>6$ months $<=1$ year & $-3.53(12)$ & $-2.69(15)$ & $-0.84(-2.29,0.61)$ \\
\hline$>1$ year $<=5$ years & $-2.85(46)$ & $-1.84(51)$ & $-1.01(-1.83,-0.19)$ \\
\hline$>5$ years & $-3.06(29)$ & $-2.87(28)$ & $-0.18(-1.27,0.91)$ \\
\hline \multicolumn{4}{|l|}{ Body Weight } \\
\hline$<=50 \mathrm{~kg}$ & $-2.83(6)$ & $-2.52(5)$ & $=0.31(-2.87,2.24)$ \\
\hline$>50 \mathrm{~kg}<=60 \mathrm{~kg}$ & $-3.47(33)$ & $-2.25(36)$ & $-1.22(-2.23,-0.21)$ \\
\hline$>60 \mathrm{~kg}<=70 \mathrm{~kg}$ & $-2.36(51)$ & $-2.70(48)$ & $0.34(-0.46,1.15)$ \\
\hline$>70 \mathrm{~kg}$ & $-2.79(37)$ & $-2.45(34)$ & $-0.35(-1.35,0.66)$ \\
\hline
\end{tabular}

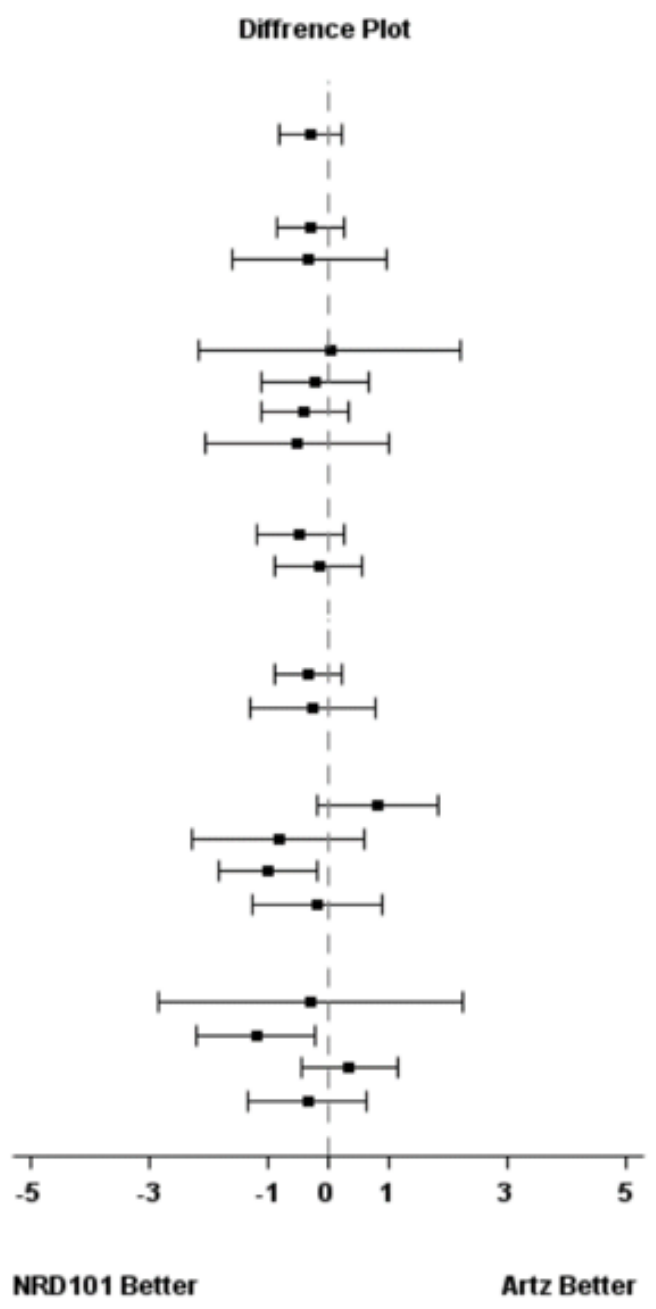

Table 5. Complications (safety analysis set) 


\begin{tabular}{|lllll|}
\hline Incidence of complications & \multicolumn{3}{l}{ NRD101 (N=131) } & \multicolumn{2}{l|}{ Artz (N=133) } \\
\cline { 2 - 5 } & Subjects & Events & Subjects & Events \\
\hline General & & & & \\
\hline AE & $34(26.0 \%)$ & 53 & $51(38.3 \%)$ & 80 \\
\hline Severe AE & $1(0.8 \%)$ & 1 & $3(2.3 \%)$ & 3 \\
\hline Serious AE & $1(0.8 \%)$ & 1 & $1(0.8 \%)$ & 1 \\
\hline Adverse reaction to study drug & $6(4.6 \%)$ & 8 & $8(6.0 \%)$ & 12 \\
\hline Serious adverse reaction to study drug & 0 & 0 & 0 & 0 \\
\hline AE leading to discontinuation of study drug & 0 & 0 & $2(1.5 \%)$ & 3 \\
\hline Death & 0 & 0 & 0 & 0 \\
\hline AE with incidence rate $\geq 2 \%$ & $1(0.8 \%)$ & 1 & $3(2.3 \%)$ & 3 \\
\hline Viral upper respiratory tract infection & $3(2.3 \%)$ & 3 & $11(8.3 \%)$ & 12 \\
\hline Upper respiratory tract infection & $6(4.6 \%)$ & 6 & $4(3.0 \%)$ & 4 \\
\hline Arthralgia & $4(3.1 \%)$ & 4 & $4(3.0 \%)$ & 4 \\
\hline Blood uric acid increased & $4(3.1 \%)$ & 4 & $1(0.8 \%)$ & 1 \\
\hline Urinary tract infection & 0 & 0 & $3(2.3 \%)$ & 3 \\
\hline Blood glucose increased & $1(0.8 \%)$ & 1 & $3(2.3 \%)$ & 3 \\
\hline Cough & 1 & & & \\
\hline
\end{tabular}

$\mathrm{AE}=$ adverse event

\section{Figures}




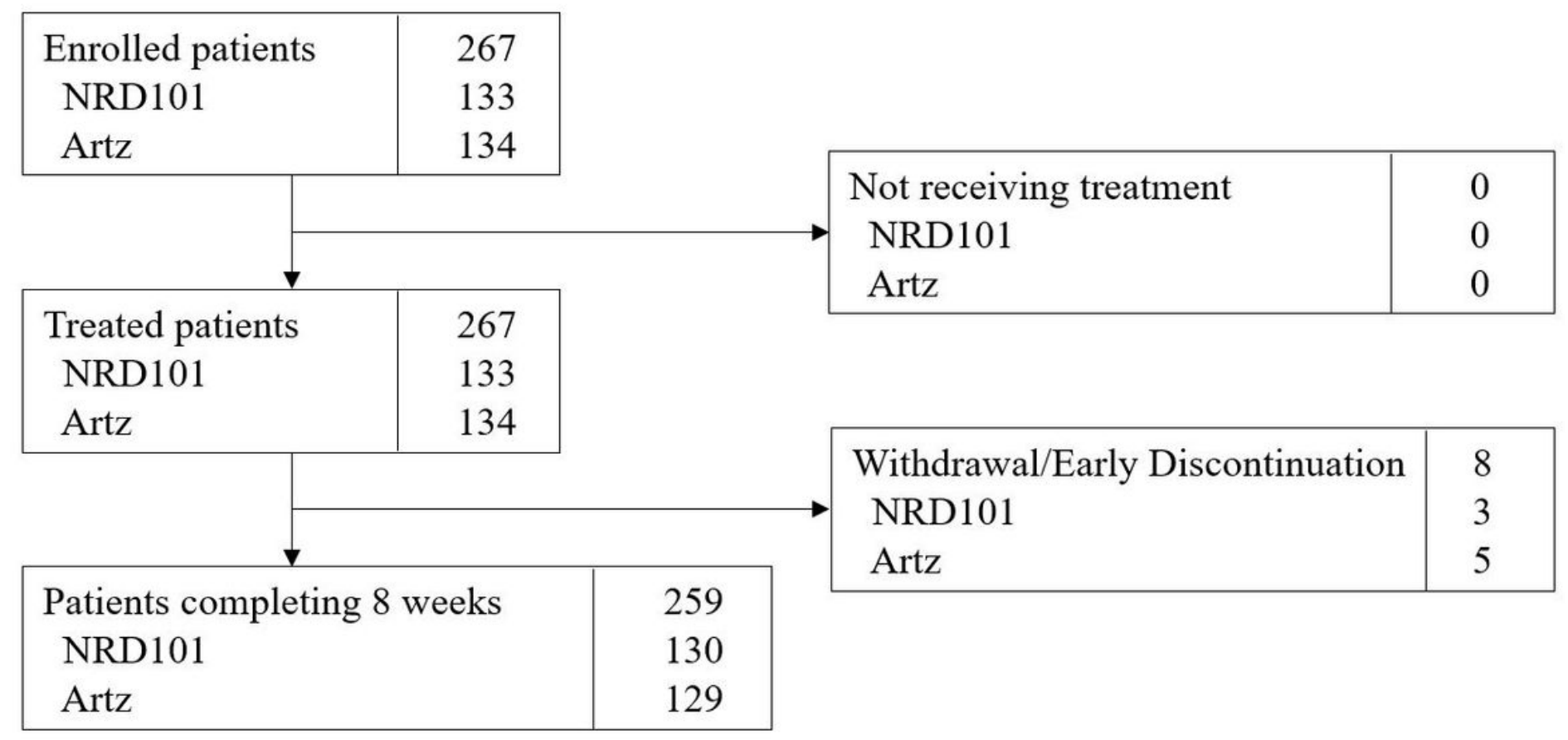

Figure 1

Disposition of patients

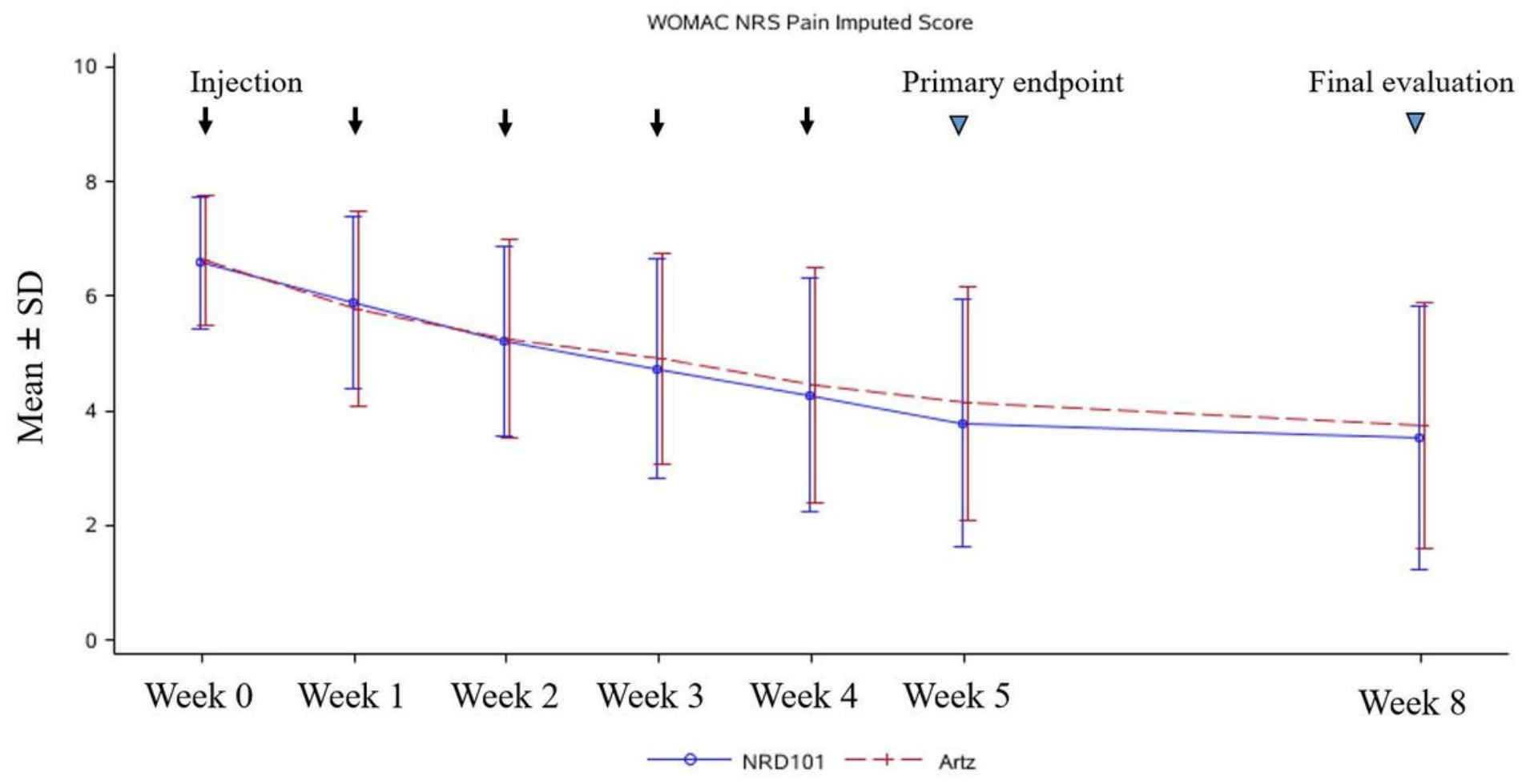

Figure 2

Transition of WOMAC NRS pain (full analysis set) 